\title{
Obstetrical Surgery in the Context of Ebola Virus Disease (Ebola) in Guinea: Lessons Learned from 2 Cases
}

\author{
Bah Elhadj Mamoudou ${ }^{*}$, Diallo Ibrahima Sory ${ }^{2,3}$, DialloThierno Saidou, ${ }^{4,5}$, \\ Leno W. Daniel' ${ }^{1}$, Soumah Aboubacar Fode Momo', Conte Ibrahima', Touré Abdoulaye4, \\ Sow Mamadou Saliou', Dao Blami ${ }^{7}$, Hyjazi Yolande ${ }^{1,7}$, Sy Telly ${ }^{1}$, Keita Namory ${ }^{1}$ \\ ${ }^{1}$ Gyneco-Obstetrics Chair, Gamal Abdel Nasser University, Conakry, Guinea \\ ${ }^{2}$ Pediatrics Chair, Gamal Abdel Nasser University, Conakry, Guinea \\ ${ }^{3}$ Institute of Nutrition and Child Health, Conakry, Guinea \\ ${ }^{4}$ Public Health Chair, Gamal Abdel Nasser University, Conakry, Guinea \\ ${ }^{5}$ Executive Secretariat/National AIDS Control Committee (ES/NACC), Conakry, Guinea \\ ${ }^{6}$ Chair of Infectious and Tropical Disease, Gamal Abdel Nasser University, Conakry, Guinea \\ ${ }^{7}$ Jhpiego,USAID, Conakry, Guinea \\ Email: *monembo12@gmail.com
}

How to cite this paper: Mamoudou, B.E. Sory, D.I., Saidou, D.T., Daniel, L.W., Momo, S.A.F., Ibrahima, C., Abdoulaye, T., Saliou, S.M., Blami, D., Yolande, H., Telly, S. and Namory, K. (2019) Obstetrical Surgery in the Context of Ebola Virus Disease (Ebola) in Guinea: Lessons Learned from 2 Cases. Open Journal of Obstetrics and $G y$ necology, 9, 1305-1314.

https://doi.org/10.4236/ojog.2019.910126

Received: June 20, 2019

Accepted: September 23, 2019

Published: September 26, 2019

Copyright ( 2019 by author(s) and Scientific Research Publishing Inc. This work is licensed under the Creative Commons Attribution International License (CC BY 4.0).

http://creativecommons.org/licenses/by/4.0/

\begin{abstract}
In this manuscript, the authors have studied obstetrical surgery in the context of Ebola virus disease in Guinea. No protocol recommends childbirth outside of Ebola treatment center, although it has no technical platform and no qualified providers in this area. These were unknown MVE cases in pregnant women/parturient women aged 25 and 40 years, with no education, who were confirmed in the RT-PCR test after surgical management. To fight Ebola virus transmission, traditional protection protocols must be strengthened. Training, supervision and monitoring of providers are key elements for the protection of staff in the event of an EVD outbreak. Improving working conditions and strengthening hand washing, usage of PPE/EPP, decontamination of equipment with $0.5 \%$ chlorine solution, hygiene of premises, immunization of personnel involved, are effective measures to combat EVD.
\end{abstract}

\section{Keywords}

Ebola Virus Disease, Childbirth, Prevention and Control of Infection, Guinea

\section{Introduction}

The Ebola epidemic in West Africa from 2014 to 2016 was the largest since the 
outbreak of the virus in Central Africa in 1976. It was so intense that there were 11.300 deaths in the 3 epidemic countries caused by the Zaire species (Ebola virus Zaire EVD). 22.4\% (Guinea), 42.6\% (Liberia) and 35.0\% (Sierra Leone). Despite this, these numbers are underestimated [1] [2].

March 2014 officially marked the beginning of the Ebola outbreak in three (3) West African countries (Guinea, Liberia and Sierra Leone). This outbreak has led to a geographical spread of the disease in Africa: (8 cases with 6 deaths) in Mali, ( 1 case) in Senegal, (20 cases with 8 deaths) in Nigeria. This spills over into other non-African countries such as the United States of America (4 cases including 1 death), the United Kingdom (1 case), Spain (1 case) and Italy (1 case) [1]. Symptoms include fever, headache, body aches, nausea, vomiting, diarrhea, bleeding, septic shock and multi-organ failure [3]. The most common mode of transmission is contact with bodily fluids from symptomatic patients [4].

Ebola virus disease (EVD) during pregnancy is often associated with a high rate of obstetric complications. Maternal and perinatal outcomes are adverse including spontaneous abortion, membranes failure before labour, premature birth, anteropartum and postpartum hemorrhage, consequently cases of death in utero are often observed. Although these are rare ones, some pregnant women have developed EVD and delivered in referral facilities in Guinea [5].

In Guinea, infection prevention and control (ICP) training were launched in February 2014 by the Ministry of Health in collaboration with USAID (Jhpiego) and other technical and financial partners to help staff protect themselves from the risk of EVD contamination. Health workers are exposed and pay a heavy price, especially doctors and nurses in public and private facilities. They are more at risk than the general population of the same age group. Although a number of protective measures associated with infection prevention training have been initiated by the implementation of personal protective measures, the late establishment of patient sorting centers at the entrance of health and safety facilities, is a step forward. A vaccination activity with the ZEBOV-GP rVSV vaccine in Conakry from March 2015 onwards, has made it possible to control transmission among staff and was only effective from July 2015 onwards [4]. Despite the fact that personal protective equipment (PPE) and caregiver protection protocols were available, individuals in the operating room and surgeons did not clearly follow instructions to perform surgery on a confirmed or suspected Ebola patient [6].

As the mode of transmission through biological fluids is not identified, only a great deal of experience will allow risk reduction and effective control of ERM.

However, some known complications of pregnancy (preeclampsia with HELLP syndrome, gestational thrombocytopenia and placental abruption) are confused with the characteristics of AEM. From this observation, the coverage must be broad [7].

Any refractive intervention in a positive or suspicious case requires a two-way analysis (utility-risk) taking into account the environment and the patient's point of view. At the end of this analysis, it is advisable to consider other thera- 
peutic modalities or even a postponement of the procedure. Faced with a failing health system, as is the case in countries affected by the epidemic, the prognosis is often bleak [7].

We report two (2) clinical cases of AEM in obstetrical settings, while discussing diagnostic challenges, caregiver exposure risks and lessons learned.

\section{First Observation}

She was received on June 30, 2015 at 7 p.m. at our service for convulsions on a pregnancy site. The gestational age was 7 months, referred by the EVD screening unit with mention of suspected Ebola cases and its temperature was $36.5^{\circ} \mathrm{C}$ on the reference receipt. According to the husband, the symptomatology began 18 hours ago, on June 30, 2015, at home, marked by a tongue biting. At her reception, she was admitted to the eclampsia room, with an obsession with consciousness. A survey of accompanying persons on the history of this pregnancy revealed that the pregnancy is not followed, the date of the last menstruation is not known and no biological or radiological evaluation conducted. The patient had an altered general state, she was agitated, the mucous membranes were well colored, anicteric, temperature at $36.8^{\circ} \mathrm{C}$, pulse rate at 90 beats per minute, blood pressure at $160 / 110 \mathrm{mmHg}$, respiratory rate at 23 cycles per minute, a physical examination revealed a bulky abdomen, an ovoid uterus with a long longitudinal axis and an upper large end. An absence of uterine contractions, a cephalic presentation of level 5/5th, the fetus back to left, the measured uterine height was $28 \mathrm{~cm}$ and the abdominal circumference $86 \mathrm{~cm}$ and an estimate of the probable weight at $2408 \mathrm{~g}+15 \%$. Noise from the fetal heart at $130 \mathrm{bp} / \mathrm{min}$. The fingertip brings back white, not foul, leucorrhoea.

In addition, we noted a discreet swelling of the lower limbs and a slight puffiness of the face. The patient had a tonic-clonic attack during the examination. Faced with this non-febrile tonic-clonic crisis with alteration of the general state, an emergency biological exploration was requested.

Within 21 days of this symptomatology, the team did not look for an epidemiological link with EVD. On arrival, the first-line blood and urine biological test showed the following anomalies: quantitative proteinuria at $3 \mathrm{~g} / 100 \mathrm{ml}$, hemoglobin level at $7 \mathrm{~g} / \mathrm{dl}$, Ts 5 minutes 23 seconds, Tc 7 minutes 35 seconds. In view of this clinical symptomatology and biological anomalies, we selected as diagnostic hypotheses: a progressive pregnancy of 30 - 32 weeks of amenorrhea in the case of a woman not in labour, with eclampsia and anemia. Behaviour adopted: taking a venous route with $0.9 \%$ saline serum, bladder catheterization bringing back $100 \mathrm{cc}$ of clear urine. Administration of $14 \mathrm{~g}$ magnesium sulfate, 4 $\mathrm{g}$ intravenously (intravenous) and $10 \mathrm{~g}$ intramuscularly (intramuscular). The patient had two (2) tonic-clonic seizures of 15 minutes, each with psychomotor agitation, which prompted a Caesarean section decision for eclampsia. She was transferred to the OR where she is placed under general anesthesia by induction with thiopental $500 \mathrm{mg}$. It was then that a transverse incision by Joël Cohen allowed by the cephalic extraction of a boy with an Apgar at the 1st minute at 5/10 
and at the 10th minute at 7/10, weighing $1800 \mathrm{~g}, 44 \mathrm{~cm}$ of height, $30 \mathrm{~cm}$ of head circumference. Administration of 2 gramm ampicillin intravenous, 10 intramuscular oxytocin intravenous, and $10 \mathrm{mg}$ diazepam intravenous. A directed delivery of the complete placenta was subsequently made possible. Thus, the parietal closure as planned was carried out and it was removed from the block at 2 hours later with the following parameters: blood pressure (BP) 130/100 $\mathrm{mmHg}$, pulse $113 \mathrm{p} / \mathrm{min}, 300 \mathrm{cc}$ of urine concentrated in the bag. The child transferred to neonatology at INSE for acute fetal suffering (AFP) and prematurity in order to ensure effective care. Less than an hour later, the patient experienced vaginal and surgical site bleeding made of fluid, in coagulated and persistent blood. There was a cooling of the extremities, cold sweat, red eyes and haggard eyes. Behavior: Implementation of a saline serum infusion with 40 UI oxytocin and oxygenation attempt in vain. The patient dies in a hypovolemic shock picture. A post-mortem sample taken on June 7, 2015 by the Red Cross was positive for MVE. The newborn transferred to neonatology for prematurity plus acute fetal suffering died after essential care. After investigations, the parents confirmed that the woman was in contact with a confirmed and followed case at Gbenty, a locality situated $50 \mathrm{~km}$ away from Forécariah and bordering Sierra Leone. She replied that she was absent as part of her follow-up on 30 June 2015 to reside in Boussoura, municipality of Matam in Conakry. Referring to the clinical context, followed as contact with epidemiological link, premature delivery, positive postmortem sampling, the patient and the newborn received a swab that allowed the diagnosis of AEM on the bodies. Thus, all the staff of the two services, 16 at the Donka maternity ward (6 doctors, 4 midwives, 1 nurse, 3 trainee students, 2 matrons) and 27 in neonatology (5 doctors, 15 nurses/technical health workers, 7 trainee students) were followed for 21 days, of which one (1) positive case was diagnosed.

\section{Second Observation}

A 25-year-old bride, saleswoman, residing at Yimbaya in the municipality of Matoto, a suburb in the Conakry. She was received on August 25, 2014 at 1 p.m. at the Matam CMC with similar symptom of the first case. She was recommended to a midwife for care at Matammunicipal medical center. Upon being received there, she was transferred to the delivery room. The symptomatology was progressive, marked by the above-mentioned signs, the persistence of fluid flow, and hyperthermia motivated a consultation for adequate management. We note in its antecedent the following: childbearing 4, parity 3. However, she did not receive any prenatal consultations (ANC) during this pregnancy. According to the parents, she left Liberia, where she was followed as a contact for a positive case of Ebola to come to Yimbaya. During this stay, no investigation relating to this symptomatology that could establish an epidemiological link with MVE was carried out. On clinical examination, the asthenic and febrile patient had the following parameters: blood pressure at $110 / 70 \mathrm{mmHg}$, temperature at $39.1^{\circ} \mathrm{C}$, Pulse 116 beats per minute. The physical examination revealed a large abdomen, an ovoid uterus with a long longitudinal axis and a large upper end. Two uterine 
contractions of 30 to 35 seconds, a cephalic presentation level 5/5th, fetal back on the right, uterine height $31 \mathrm{~cm}$, abdominal circumference at $90 \mathrm{~cm}$ and an estimate of the probable weight at $2790 \mathrm{~g}+15 \%$. Fetal heart sounds at 102 beats per minute. The vulva coated with liquid of greenish appearance, the vagina was warm to the touch, and the cervix was posteriorly open to a finger at both openings. Its lower segment well amplified on the fetal head, a mobile cephalic presentation, and the fingering has brought a foul greenish liquid flow. In response to the symptoms elucidated by the diagnosis of active pregnancy of 36 - 37 weeks of amenorrhea, a 48-hour premature rupture of the membranes with probable chorioamniotitis and fetal suffering (FS) was established. The indication of a cesarean section for FAS in the first line of the labour phase was indicated. Actions carried out: Taking a venous route with a lactate Ringer solution, $2 \mathrm{~g}$ Ampicillin IVL. She was transferred to the operating room at 11:20 am, the placement of a bladder probe collected $180 \mathrm{cc}$ of dark, non-hematic urine. The team had not previously received training in infection prevention and control and neither had it provided the necessary means of protection, namely: personal protective equipment/preventive protective equipment, or double gloving. After a transverse incision by Joël Cohen, cephalic extraction at 11:25 am from an Apgar boy at the 1st minute $6 / 10$ and at the 5th minute 8/10; weight: $2800 \mathrm{~g}$; height: $52 \mathrm{~cm}$; head circumference: $32 \mathrm{~cm}$. Injection of $10 \mathrm{IU}$ in IVL and $10 \mathrm{IU}$ of oxytocin into the serum was done. The directed delivery of the complete placenta was effective. A hysterography with vicryl crimped 1 was performed and homeostasis was conducted as well. The plan-by-plan parietal closure with vicryl set 2 on the aponeurosis and the skin in separate point was carried out. The child after resuscitation developed petechiae and was transferred to Donka's neonatology department, where he died on survival day 1 . The patient was transferred to the recovery room with an infusion containing 10 UI of oxytocin, where she developed immediate postpartum hemorrhage (PPH) by uterine inertia at 14:20 minutes. The following procedures were performed: uterine massage, compression of the abdominal aorta, administration of metergin $0.5 \mathrm{mg}$ IM. The persistence of bleeding and fluidity required the request and transfusion of two $\mathrm{O}+$ blood bags and at 15:18 minutes. The patient was readmitted to the operating room for a hysterectomy and hemostasis was performed. Despite the conducted hysterectomy and transfusion, the bleeding persisted with fluid and uncoagulated blood on the stitches. The patient died in a hypovolemic shock picture. Considering the clinical context of fever, diarrhea, bleeding, and unreported stay in Liberia to cause response teams to take a sample that tested positive for EVD, his care made 10 direct contacts among the staff ( 3 doctors, 4 midwives, 1 nurse, 2 student interns), of whom 5 were confirmed RT-PCR test-positive conducted and followed at the Donka Ebola Treatment Centre, out of which two (2) doctors died (Table 1, Figure 1).

\section{Discussion}

During the period from August 2014 to November 2015, two parturient women 
Table 1. Summary of two (2) clinical cases observed.

\begin{tabular}{|c|c|c|}
\hline Maternal monitorings & Observation 1 & Observation 2 \\
\hline \multirow{5}{*}{$\begin{array}{l}\checkmark \text { Socio-demographic statuts } \\
\checkmark \text { Places visited }\end{array}$} & 40 years & 25 years \\
\hline & Gestity: 11; parity: 10; number of living children: 6 & Gestity: 3 ; parity: 3 \\
\hline & Household & Saleswoman \\
\hline & Married; place of residence: Matam in Conakry & Married; place of residence: Matoto in Conakry \\
\hline & Moves to Liberia and comeback to Matam & Forécariah and Yimbaya \\
\hline$\checkmark$ Clinical signs at reception & - Temperature: $36.5^{\circ} \mathrm{C}$; valided; Eclampsia & AFS. Caeserean (30 mn $05 \mathrm{~s})$ \\
\hline$\checkmark$ Diagnostic & - Caesarean (intervention duration 37 minutes) & Homeostasis hysterectomy (1 hour $11 \mathrm{mn}$ ) \\
\hline $\begin{array}{l}\text { Mode of delivery, } \\
\text { Complications }\end{array}$ & $\begin{array}{l}\text { Postpartum hemorrhage with cardiovascular shock } \\
\text { and hypovolemic shock }\end{array}$ & $\begin{array}{l}\text { Postpartum hemorrhage with cardiovascular and } \\
\text { hypovolemic shock }\end{array}$ \\
\hline
\end{tabular}

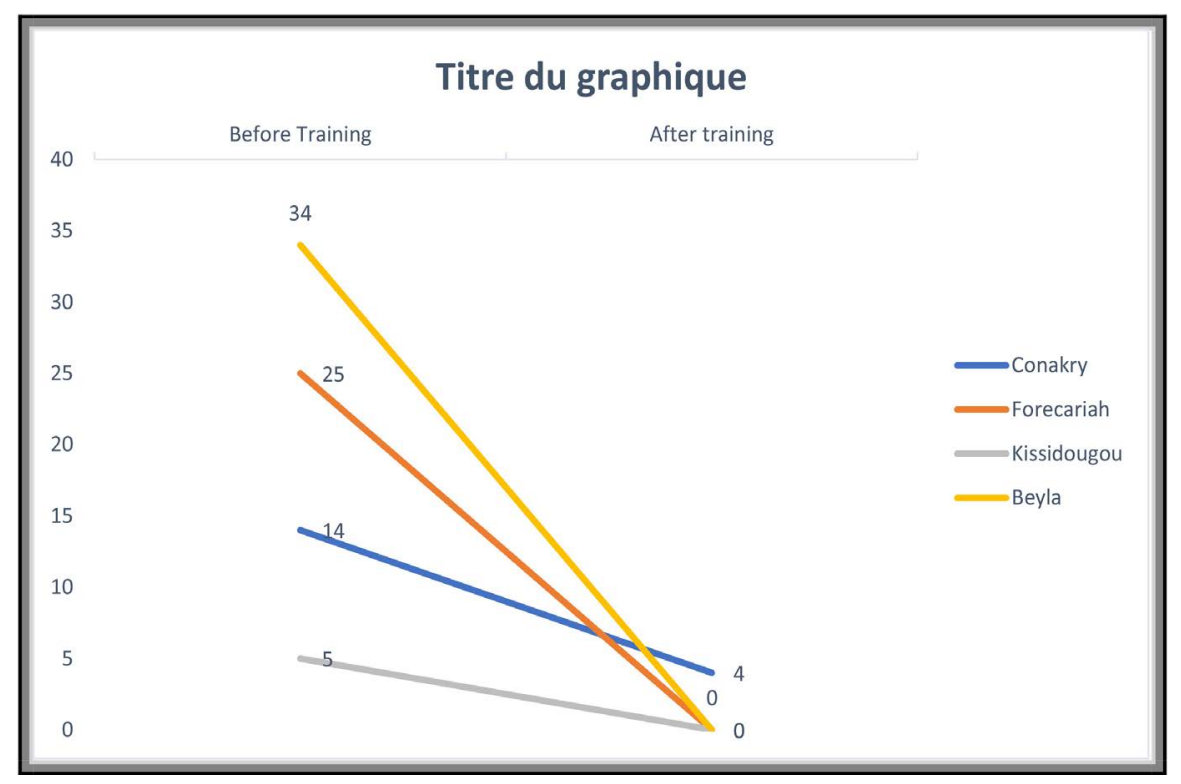

Figure 1. Evolution of personal contamination by EBV before and after IPC training.

were confirmed with EVD after cesarean deliveries in two maternity hospitals in Conakry city (the Donka National Maternity Hospital and the Matam Municipal Medical Centre). These were places of deliveries for acute fetal suffering complicated by hemorrhagic shock, with hysterectomy and homeostasis performed at the Matam Municipal Medical Centre. Eclampsia was also complicated by hemorrhagic shock. The personnel involved was followed for contact before the ICP training (6 confirmed cases including 3 deaths).

Pregnancy promotes a state of progressive immunotolerance that inhibits fetal rejection; this may have hindered pyrogenic reactions. EVD is complex and is supposed to be at least partially immunodependent. The unique immunological status of pregnant women may affect the presentation and progression of the disease. This highlights the challenges that clinicians can face in assessing pregnant women for possible infections, and the potential risk to health personnel [8]. Until here, there is not any treatment for this disease, but the prevent vaccine can protect those people who has been contacted. 
Status of infection prevention and control (IPC) training for health care providers: (Before and after the training).

\subsection{Before Infection Control and Prevention Training}

There were two (2) clinical cases, ten (10) providers were exposed to maternity and followed up for contact for 21 days (including 5 confirmed cases including 2 deaths at the Ebola treatment centre). The two deceased were both doctors. For, the newborn was transferred to neonatology and hospitalized in an intensive care unit, 7 contacts were identified (no contamination). The two surviving physicians recounted this, and I quote.

I helped my colleagues perform a hemostasis hysterectomy after the failure of the B-Lynch technique in a cesareanized woman who was still bleeding. I had not done the double gloving and my PPE was not protecting me properly, I felt that I was exposed to blood and body fluids. After the surgery, I washed my hands and faced with plenty of water. We were informed a week after this operation that the woman had Ebola. I had my first symptoms on the 15th day at home, marked by fever $\left(38.07^{\circ} \mathrm{C}\right)$, physical asthenia, cold and rhinorrhea. I went to Donka's ETC on 9/9/2014 at 10:00 am, I was sampled and my RT-PCR test was positive. Fever, diarrhea, vomiting, physical asthenia, are the symptoms that made me tired. The resident who was in charge of supervision was already hospitalized at the ETC and died on the 10th day of his admission. The one (the doctor) who had done the cesarean section was willing to come to the ETC, she also died on the 6th day of her admission. I left the CTE on the 18th day of my admission. I am now a national trainer in infection prevention and control, I have participated on behalf of (Jhpiego) in the training of national trainers and health providers.

\subsection{After the Training in Infection Prevention and Control}

Clinical observation (3 and 4) 67 providers were followed for direct contact and none developed the disease. Out of 99 direct contacts recorded at the Maternity Hospital, 6 maternal health providers were infected (6.06\%) and 2 deaths (2\%). In neonatology, 34 providers were overall contact subjects (clinical observation 1 and 2). The contacts of the four reported cases were subject to post-exposure stigmatization and followed up for 21 days of stress. Non-contagion would be linked to the cascade training of ICH organized by the Ministry of Health of Guinea, with technical and financial support from USAID (Jhpiego-Guinea) since December 2014, and the application of WHO ICH guidelines based on the sharing of standard precautions and transmission mode precautions. Some of them had successfully received experimental Ebola vaccination in Guinea [5] [6].

Of all confirmed and suspected cases recorded in the country, 211 concerned health professionals, 115 of whom died (54.5\%). From January to July 2015, the Sonfonia Treatment Centre cared for 26 health professionals, 18 of whom came out cured (69.2\%). Physicians and nurses were the most infected overall (49\% of infected health professionals), as well as technical health workers (18\%). Health 
professionals were, with an incidence rate of 21.5 per 1000 , nearly 48 times more infected than the rest of the population aged 15 years and over, whose rate was 0.45 per 1000 [1].

Maternity care providers are exposed to blood and body fluids on a daily basis. Attempts to reduce the "medicalization" of childbirth have led to poor infection control practices, resulting in poor coating and sometimes skin of health care providers that can be contaminated with blood, amniotic fluid and feces. Although such contaminants may contain pathogens such as hepatitis C or HIV (or other agents not yet identified), the wearing of impermeable surgical clothing and protective glasses does not generally appear to be a major concern.

In Guinea, maternity care providers are exposed daily to blood and body fluids. Attempts to reduce the "medicalization" of childbirth have led to poor infection control practices, resulting in poor coating and sometimes skin of health care providers that can be contaminated with blood, amniotic fluid and feces. Although such contaminants may contain pathogens such as hepatitis C or HIV (or other agents not yet identified), the wearing of impermeable surgical clothing and protective glasses does not generally appear to be a major concern. Caregivers should never be exposed to their patients' blood or body fluids wherever they work. The same observation has been made in developed countries such as Canada [3].

Obstetric care providers should ensure that they have received training in the wearing and removal of PPE and that they comply with the recommended guidelines (including those dealing with proper training and supervision in the use of PPE). There is good reason to hope that the fear of EVDs will eventually ease in the same way. This will provide the full range of intensive and surgical care for patients infected with Ebola virus. The American College of Surgeons [7] has adapted the U.S. Centers for Disease Control and Prevention guidelines for surgical procedures in patients suspected of having Ebola virus disease. Surgeons should review these guidelines frequently for updates. Currently, the guidelines do not provide any information on patients who should undergo surgery.

When emergency intervention or surgery is being considered for a patient with Ebola disease, the health care team should conduct a risk and utility analysis, which should take into account not only the patient's perspective, but also the broader environment. Other therapeutic modalities and the postponement of the intervention, should be the preferred choices for the time being. Full approval of generally-accepted oral rehydration methods should be encouraged rather than parenteral rehydration and peripheral administration rather than central venous administration. The number of blood tests should be reduced as much as possible and limited to those performed in the health environment.

The number of imaging tests should also be significantly lower in patients with EVD than in unaffected patients. The implantation of arterial catheters will be very difficult to justify. To reduce the risk of direct patient contact, an automated and non-disruptive method of monitoring blood pressure and oxygen sa- 
turation should be used [7].

\section{The Lesson Learned about 2 Cases}

- Suspect or confirmed cases must be managed at the ETC. In case of suspicion of MVE, no invasive action should be taken outside the Ebola treatment centre, to prevent the spread of the disease.

- There is no protocol or guideline that recommends surgery for EVD.

- This is the need to train all staff in ICH in general, screening and screening of patients specifically and monitoring as well as evaluation of structures and staff.

- Consider everyone as potentially infected, or likely to be infected by us. This is the golden rule of standard precautions and precautions based on the transmission of infections in a health facility. During the outbreak of EVD, it can be reinforced by screening and sorting.

- The systematic taking of temperature, allows detecting cases of fever but its absence does not exclude the EVD, especially for the case of a pregnant woman.

- Reluctance of the population to manage EVD, due to poor communication, a breakdown in trust between the population and caregivers, or the first information received about the disease and lack of knowledge of the disease (a little-known and imported disease).

\section{Conclusion}

No protocol recommends childbirth outside of Ebola treatment center, although it has no technical platform and no qualified providers in this area. These were unknown MVE cases in pregnant women/parturient women aged 25 and 40 years, with no education, who were confirmed in the RT-PCR test after surgical management. To fight Ebola virus transmission, traditional protection protocols must be strengthened. Training, supervision and monitoring of providers are key elements for the protection of staff in the event of an EVD outbreak. Improving working conditions and strengthening hand washing, usage of PPE/EPP, decontamination of equipment with $0.5 \%$ chlorine solution, hygiene of premises, immunization of personnel involved, are effective measures to combat EVD.

\section{Contributions of the Authors}

All authors participated in the realization of the study, read and approved the final version.

\section{Acknowledgements}

The authors thank UGANC, USAID (Jhpiego-Guinea), WHO-Guinea, who technically and materially supported this study.

\section{Conflicts of Interest}

The authors declare no conflict of interest. 


\section{References}

[1] Migliani, R., et al. (2016) Maladie à virus Ebola. Médecine et Armées, 44, 149-160.

[2] Organisation Mondiale de la Santé (2015) Recommandations Provisoires: Maladie à virus Ebola pendant la grossesse: Dépistage et prise en charge des cas d'Ebola, des contacts et des survivants.

[3] Money, D., et al. (2015) Lignes directrices quant à la prise en charge au Canada d'une femme enceinte ayant été exposée à la maladie à virus Ebola ou ayant été infectée par ce virus. Journal of Obstetrics and Gynaecology Canada, 37, 190-191. https://doi.org/10.1016/S1701-2163(15)30341-8

[4] Bah, E.I., et al. (2015) Clinical Presentation of Patients with Ebola Virus Disease in Conakry, Guinea. The New England Journal of Medicine, 372, 40-47. https://doi.org/10.1056/NEJMoa1411249

[5] Lado, M., et al. (2015) Clinical Features of Patients Isolated for Suspected Ebola Virus Disease at Connaught Hospital, Freetown, Sierra Leone: A Retrospective Cohort Study. The Lancet Infectious Diseases, 15, 1024-1033. https://doi.org/10.1016/S1473-3099(15)00137-1

[6] Safari, J. (2015) Insuffisance rénale aiguë des patients atteints du virus hémorragique Ebola au centre de traitement de Macenta en Guinée Forestière. Néphrologie \& Thérapeutique, 11, 385. https://doi.org/10.1016/j.nephro.2015.07.367

[7] McAlister, V. (2014) La chirurgie chez le patient atteint de la maladie à virus Ebola. Canadian Journal of Surgery, 57, 366. https://doi.org/10.1503/cjs.016014

[8] Safari, J., et al. (2015) Insuffisance rénale aiguë des patients atteints du virus hémorragique Ebola au centre de traitement de Macenta en Guinée Forestière. Néphrologie \& Thérapeutique, 11. 385. 